\title{
Médiévales
}

Langues, Textes, Histoire

52 | printemps 2007

Le livre de science, du copiste à l'imprimeur

\section{Aspects du discours normatif dans le Roman de Tristan en prose (coutumes, codes sociaux, conversation)}

Olivier Linder

\section{(2) OpenEdition}

Journals

Édition électronique

URL : https://journals.openedition.org/medievales/2573

DOI : $10.4000 /$ medievales. 2573

ISSN : $1777-5892$

Éditeur

Presses universitaires de Vincennes

Édition imprimée

Date de publication : 1 juin 2007

Pagination : 153-170

ISBN : 978-2-84292-202-3

ISSN : 0751-2708

Référence électronique

Olivier Linder, «Aspects du discours normatif dans le Roman de Tristan en prose (coutumes, codes sociaux, conversation) », Médiévales [En ligne], 52 I printemps 2007, mis en ligne le 06 septembre 2009, consulté le 22 avril 2022. URL : http://journals.openedition.org/medievales/2573 ; DOI : https:// doi.org/10.4000/medievales. 2573 


\section{Olivier LINDER}

\section{ASPECTS DU DISCOURS NORMATIF DANS LE ROMAN DE TRISTAN EN PROSE (coutumes, codes sociaux, conversation)}

Dans l'histoire des mentalités et des mœurs médiévales coexistent deux représentations plus ou moins antithétiques. D’une part, un Moyen Âge « violent, sale et méchant ${ }^{1}$ » où balbutient à peine la «civilisation des mœurs » et la « curialisation des élites ». D'autre part, un Moyen Âge très réglé, où la pression normative s'exerce avec force ${ }^{2}$. Cette ambivalence exprime au moins une réalité : il n'existe pas de société qui ne connaisse de réflexion sur la norme sociale. Selon les définitions usuelles, les normes sont des modèles culturels de conduite adoptés par un groupe ou une société, légitimés par des valeurs partagées jusqu'à un certain point, et dont les mours sont la dimension pratique ${ }^{3}$. Elles nécessitent un apprentissage, marquent l'adhésion à des valeurs communes, fondent l'appartenance au groupe. Leur transgression ou méconnaissance entraîne des sanctions, réelles ou symboliques. Du fait de l'écart entre "ce que nous sommes censés faire" et "ce qu'en réalité nous faisons", elles connaissent un phénomène de régulation permanente.

Nous aborderons ici les normes induites par l'idéologie aristocratique telle que la reflète et la construit le roman de chevalerie, à l'exemple du Roman de Tristan en prose ${ }^{4}$. Divers aspects du Tristan en prose ${ }^{5}$ le désignent comme un

1. Cf. R. Muchembled, L'Invention de l'homme moderne. Cultures et sensibilités en France du XV ${ }^{e}$ au XVII siècle, Paris, 1988, chap. 1.

2. Cf. l'important article de B. CAzELLES, «Outrepasser les normes : l'invention de soi en France médiévale », dans K. BrownleE et S. G. Nichols dir., Boundary and transgression in medieval culture (Stanford French Review, XIV), 1990, p. 69-92.

3. Cf. R. Boudon et F. Bourricaud dir., Dictionnaire critique de la sociologie, Paris, 1982 ; A. Akoun et A. Ansart dir., Dictionnaire de sociologie, Paris, 1999.

4. Sur l'empreinte de l'idéologie aristocratico-chevaleresque dans le roman médiéval, $c f$. entre autres A. Scaglione, Knights at Court. Courtliness, Chivalry and Courtesy from Ottonian Germany to the Italian Renaissance, Berkeley, 1991. Sur son empreinte dans le Tristan en prose, cf. notre thèse de doctorat: Le jeu sérieux. La représentation idéalisée de l'univers aristocratique dans le "Roman de Tristan" en prose, Université de Nancy II, 2006.

5. Dans les trois éditions disponibles : R.-L. Curtis éd., 3 vol., Munich, Leyde, Woodbridge, 1963-1985 (début du roman) ; version II dite "Vulgate", Ph. MÉnARD dir., 9 vol., Paris, Genève, 1987-1997; version I d'après le ms. BnF 757, Ph. MÉnARD dir., 4 vol., Paris, 1997-2003. De 
support privilégié pour une telle approche. En effet, alors même qu'il recycle des récits antérieurs dont la portée transgressive est connue ${ }^{6}$, le Tristan en prose procède à leur «banalisation aristocratique $»^{7}$. L'adultère des héros est justifié par la dégradation du roi Marc ${ }^{8}$, et surtout par la courtoisie dont Tristan et Yseut sont des modèles accomplis. Quant au code courtois, le Tristan en prose se place donc résolument du côté de la conformité et non de la déviance. Le discours normatif y est considérablement amplifié : conformément à la technique narrative de la plupart des romans en prose, le récit principal est fragmenté en une multitude de micro-récits stéréotypés et exemplaires : joutes, défenses de ponts, haltes nocturnes, conversations, plaintes d'amour, etc. ${ }^{9}$.

Ce phénomène, plus envahissant que dans Lancelot en prose, a généré un sentiment de "vide" idéologique chez nombre de lecteurs, depuis J. Bédier - qui parlait sans aménité d'un «fatras d'inventions chevaleresques » - jusqu'à E. Baumgartner pour qui le roman résonne du «vain bruit des combats » et qui remarque que l'Aventure, concept central du roman de chevalerie, y paraît bien souvent vide de sens ${ }^{10}$. De notre point de vue, ce "vide" est comblé par un discours normatif omniprésent, exclusivement orienté vers les relations de sociabilité, extrêmement ritualisées, entre les membres de la caste aristocratique. Un étroit réseau de prescriptions fait du chevalier un être dont la distinction ${ }^{11}$ est

façon générale, $c f$. les ouvrages d'E. BAUMGaRTner, Le "Tristan en prose". Essai d'interprétation d'un roman médiéval, Genève, 1975 et La Harpe et l'Épée. Tradition et renouvellement dans le "Tristan en prose", Paris, 1990.

6. Sur la subversion sociale et morale dans les Tristan en vers, $c f$. par exemple J. SubREnAt, «Sur le climat social, moral, religieux du Tristan de Béroul », Le Moyen Âge, 82, 1976, p. 219261 ; J.-Ch. PAYEN, «Ordre moral et subversion politique dans le Tristan de Béroul», dans Mélanges J. LoDS, Paris, 1978, t. I, p. 473-484 ; B. N. SARGENT-BAur, «Between Fabliau and Romance : Love and Rivalry in Beroul's Tristan », Romania, 105, 2-3, 1984, p. 292-311 ; MarieLouise OlLIER, «Le statut de la vérité et du mensonge dans le Tristan de Béroul », Tristan et Iseut, mythe européen et mondial. Actes du colloque des 10, 11 et 12 janvier 1986, D. BUSCHINGER éd., Göppingen, 1987, p. 298-318.

7. Cf. J. Kooljman, « À propos du Tristan de Béroul et du Tristan en prose », Romanische Forschungen, 91, 1979, p. 96-101.

8. $C f$. F. Wiseman-Wiedemann, «From Victim to Villain : King Mark », dans N. B. Smith et J. T. SNow éd., The Expansion and Transformation of Courtly Literature, Athens (Ga), 1980, p. 49-68 et B. Milland-Bove, "Les aventures de Marc en Logres dans le Tristan en prose», Bien Dire \& Bien Aprandre («Figures du roi »), 17, 1, 1999, p. 73-83.

9. Pour un essai de typologie de ces séquences, $c f$. E. BAumGartner, Le Tristan en prose..., $4^{\mathrm{e}}$ partie, chap. II, «Structures romanesques », p. 273-283, et La harpe et l'épée..., p. 25-26.

10. Cf. La harpe et l'épée..., p. 52: "le récit se heurte à l'in-signifiance de l'aventure, voire à son absence ». Le jugement de J. Bédier est rapporté par E. BAUMGarTnER, Le Tristan en prose..., p. IX.

11. Sur cette notion essentielle, $c f$. l'article «Distinction» du Dictionnaire raisonné de la politesse et du savoir-vivre du Moyen Âge à nos jours, A. MonTANDON dir., Paris, 1995 (p. 271303). Condition de la visibilité sociale, elle implique «séparation, supériorité et élégance. » Elle se manifeste par un code des manières qui permet d'asseoir le respect des hiérarchies. Paradoxalement, elle implique à la fois conformisme et singularité (p. 272). Cf. B. CAZELLES, loc. cit., p. 72. Cf. aussi P. Bourdeu, La distinction. Critique sociale du jugement, Paris, 1979, selon qui (en substance) il faut faire "ce qui se fait" pour montrer "qui l'on est" à l'intérieur de sa caste. Pour Cicéron déjà (De officiis), le decus - le comportement convenant à la dignité sociale - s'impose au vir bonus désireux d'acquérir l'honestas morum. Sur la postérité médiévale de cette doctrine, $c f$. C. S. JAEGER, The Origins of Courtliness. Civilizing Trends and the Formation of Courtly Ideals (939-1210), Philadelphie, 1985. 
assurée précisément par la contrainte, dans l'idée que "noblesse oblige ${ }^{12}$ ". Un sens aigu de la convenance régit le comportement du chevalier : situations, attitudes, actes, gestes ou paroles strictement codifiés révèlent en lui l'aristocrate ${ }^{13}$. La critique a d'ailleurs déjà souligné cette dimension du Tristan en prose ${ }^{14}$, dont la spécificité nous paraît être une certaine fascination pour l'énoncé normatif.

Même si le roman de chevalerie véhicule dès l'origine un discours sur les valeurs de l'aristocratie ( $c f$. le début de l'Yvain ou les dilemmes de Lancelot dans le Chevalier à la Charrette), une particularité du Tristan en prose est, de notre point de vue, la complexité du discours normatif : les normes y sont pléthoriques, les choix de conduite rarement binaires, les personnages impliqués souvent nombreux. Le code de conduite est en voie d'élaboration permanente. Par exemple, la joute obligatoire avec tout chevalier de rencontre est une norme propre au royaume de Logres et à la chevalerie errante. Elle a pour fonction de départager les bons des mauvés, donc d'établir une hiérarchie. Elle n'est « vaine » ou «vide» qu'en apparence, car les combattants y gagnent ou y perdent leur honneur, notion capitale pour un public médiéval foncièrement différent du public moderne, parce qu'il appartenait à une société où l'idée d'aristocratie - au sens étymologique de "domination dévolue aux meilleurs" - était acceptée comme normale (c'est-à-dire normative). La joute est un instrument de distinction, c'est-à-dire une figuration littéraire du discours normatif. Le centre d'intérêt premier du récit n'est plus l'Aventure (qui confronte le chevalier à l'Autre dans le but d'un perfectionnement individuel), mais "les autres", 1'"être-ensemble" et ses règles. Dans ce cercle restreint, la perspective qui hante le chevalier n'est plus tant l'échec face à l'Aventure que la dérogeance par rapport à ses pairs.

On s'interrogera sur les modes d'inscription de la norme dans un texte de fiction ${ }^{15}$. Comment la fiction chevaleresque se fait-elle normative ? À l'inverse, comment l'énoncé de la norme chevaleresque produit-il un texte pleinement littéraire plutôt que simplement didactique ? On ne proposera ici que quelques pistes d'analyse dans trois domaines : les coutumes arthuriennes, les codes qui régissent les interactions sociales, enfin la conversation.

12. Une constante de la mentalité aristocratique désigne l'homme de bien comme celui qui s'impose une "discipline consentie » que le roturier ignore et ne peut comprendre, et qui lui sert à se définir et à reconnaître ses pairs. Cf. E. Mension-Rigau, Aristocrates et grands bourgeois, Paris, 1991, p. 234-298 (sur l'aristocratie moderne).

13. Les textes ne manquent pas, qui soulignent la nécessaire coïncidence entre l'être et les attentes sociales : cf. Lancelot do Lac (E. KenNEDY et F. Mosès éd., Paris, 1991, p. 138) : [Lancelot incognito] se savoit si belement tenir en lor compagnie [celle des nobles jeunes gens] que tuit cil qui le veoient cuidoient que il fust uns des plus gentis hom do monde, et pour voir si estoit il.

14. Cf. J. LaRmat, «Le Roman de Tristan en prose, manuel de courtoisie », dans E. Ruhe et R. SCHWADERER éd., Der altfranzösische Prosaroman. Funktion, Funktionswandel, und Ideologie am Beispiel des "Roman de Tristan en prose." Kolloquium Würzburg, 1977, Munich, 1979, p. 46-67.

15. Par opposition aux ouvrages de "didactique chevaleresque" ou les nombreux "traités des manières" (éthique chevaleresque et savoir-vivre étant souvent réunis dans une même chaîne valeurs-normes-mœurs). $C f$. Cl. Roussel, «Le legs de la rose. Modèles et préceptes de la sociabilité médiévale », dans A. MonTANDON dir., Pour une histoire des traités de savoir-vivre en Europe, Clermont-Ferrand, 1994, p. 1-90. Cf. aussi l'historien M. KeEN, Chivalry, Yale, 1984, p. 4-17 et 148-162. 


\section{Coutumes}

On connaît le rôle de la coutume comme élément structurant de l'univers romanesque arthurien ${ }^{16}$. Fréquente dans le Tristan en prose, elle est une norme "institutionnelle" qui se superpose aux normes habituelles, morales ou sociales. Indiscutable, elle s'impose au chevalier parce qu'il est chevalier ${ }^{17}$, et manifeste son élection héroïque. Il peut s'agir d'un pont à passer au prix d'une joute ( $c f$. infra), ou encore d'un château où le gîte se mérite à la pointe de la lance (v. II, t. II, § 39 sq.), ou plus simplement d'un comportement prédéfini par une "institution": la Table Ronde, le royaume de Logres, ou la chevalerie errante ${ }^{18}$.

Un cas exemplaire met en scène Lancelot, Keu d'Estraus et Brandelis devant un pont gardé par Neronneus :

«Signeur cevaliers aventureus, autre passage querés que cestui se vous a moi ne volés jouster, car bien saciés certainnement que quitement vous ne poés cest pont passer !» (v. II, t. I, § 5)

Keu d'Estraus s'élance et est abattu. Il requiert le droit de poursuivre le combat a outrance, mais l'adversaire décline sa demande en vertu d'une clause spéciale :

«La coustume de cest pont n'est mie tele que je me combate a cevalier puis que je l'ai abatu. » (ibid.)

Il lui autorise donc le passage,

« car d'ore en avant ne le poés vos passer sans honte. » (ibid.)

Le paragraphe suivant réitère la scène avec Brandelis, également désarçonné. Neronneus alors

s'en retourne esranment ariere et s'arreste de cele part u il soloit estre quant il le pont gardoit, et dist adont as deus cevaliers : "Signeur cevalier aventureus, or poés le pont passer a tele hounour com vous veés : honni et vergogné le passerés, ce poés veoir apertement. » (v. II, t. I, § 6)

Quand Brandelis demande à engager la bataille, il lui est répondu que

« la coustume de cest pont est tele que s'il avenoit par aucune aventure que nous nous entrabatissom ensamble, dont i seroit la bataille, s'il voloit ; mais autrement non, che saciés. » (ibid.)

16. Cf. la thèse de S. Boumrar, La coutume dans les romans de chevalerie en France au Moyen Âge (XII ${ }^{e}$-XIII ${ }^{e}$ s. ) : étude historique, anthropologique et littéraire, Université de Paris-IV Sorbonne, 2004.

17. Les coutumes ne s'appliquent pas aux écuyers (v. II, t. I, § 7-9; cf. infra).

18. En décidant d'arborer deux épées (Curtis, I, § 322), Palamède se soumet à l'obligation éventuelle d'affronter deux adversaires simultanément, d'après la costume del reaume de Logres selon Gauvain, qui précise : «Par ce conoist l'en les chevaliers parfaiz». 
Les deux personnages n'ont plus qu'à ravaler leur honte,

por ce k'il sevent bien k'il ne pueent par raison faire force de bataille au cevalier du pont. (ibid.)

La coutume détermine mécaniquement la conduite des personnages comme en témoignent l'impeccable "chorégraphie" équestre de Neronneus et l'énoncé sentencieux des conditions attachées à la défense du pont. La coutume implique le respect aveugle d'une procédure immuable : la remarque finale montre assez que les chevaliers s'y soumettent par principe. Une fois acquises quelques précisions subsidiaires, plus rien ne peut être modifié. Il n'y a "aucun motif rationnel" ("il ne pueent par raison") pour forcer le chevalier à livrer passage. Soumission étrange : rien n'empêcherait d'engager le combat contre la volonté de Neronneus... Mais "faire force" à un chevalier et à la coutume semble une transgression trop grave pour être envisagée.

De fait, la gratuité formelle de la coutume s'efface devant sa finalité. Le passage du pont détermine une profonde modification de l'être des chevaliers: "honnis et vergognés" par la défaite, Keu et Brandelis ont en quelque sorte changé de nature. On notera le vocabulaire très présent de l'honneur, et la tournure rituelle des paroles proclamant la honte des vaincus. Or, dans une société "traditionnelle" telle que la société médiévale, la question de l'honneur est loin d'être de pure forme. En effet,

«L'honneur est la valeur que la personne possède à ses propres yeux, mais c'est aussi ce qu'elle vaut au regard de ceux qui constituent sa société. C'est le prix auquel elle s'estime [...] en même temps que la confirmation de cette revendication par la reconnaissance sociale de son excellence [...]. L'honneur implique non seulement qu'on se conduise d'une certaine façon, mais qu'en retour on bénéficie d'un traitement particulier. [...] Qui prétend à l'honneur doit se faire accepter conformément à l'idée qu'il se fait lui-même de sa propre valeur. » ${ }^{19}$

Du fait même de son aspect conventionnel, la coutume permet la vérification et la publication de l'honneur ou de l'infamie. Dès le Lancelot de Chrétien de Troyes, la coutume de la charrette n'avait pas d'autre but que d'introduire une réflexion sur l'honneur et sa relativité ${ }^{20}$, débat qui traverse une bonne part de la littérature chevaleresque ${ }^{21}$.

La suite du passage pousse à son paroxysme la logique de la coutume. Resté en retrait, Lancelot est à présent face à un dilemme. Il a promis au père de Neronneus de ne pas lever la main sur son fils :

19. J. Pitt-Rivers, Anthropologie de l'honneur, Paris, 1997 (1 $1^{\mathrm{re}}$ éd., 1977), p. 18.

20. Cf. E. J. MiCKEL, «The Theme of Honor in Chrétien's Lancelot», Zeitschrift für romanische Philologie, 91, 3/4, 1975, p. 243-272.

21. Sur l'honneur comme valeur fondatrice et principe narratif, $c f$. Ph. ConTAmine, «Honneur et chevalerie : l'enracinement médiéval », Séance publique annuelle des cinq académies, mardi 22 octobre 2002, Paris, Palais de l'Institut, 2002. Cf. encore M. MANCINI, « Onore cavalleresco e onore aristocratico », L'immagine riflessa, 12, 1989, p. 147-192, et Y. RoBREAU, L'honneur et la honte. Leur expression dans les romans en prose du "Lancelot-Graal" (XII ${ }^{e}$-XIII ${ }^{e}$ s.), Genève, 1981. 
Se il a cest cevalier se prent, dont fausera il au pere de son couvenent, si fera trop grant vilonnie ; et se il la honte de ces deus chevaliers ne venge, ki sont si compaingnon de la Table Reonde, il se mesfait trop durement. (v. II, t. I, § 7)

Lancelot est contraint de choisir entre deux injonctions normatives, qui reposent sur une même donnée axiologique : l'un ou l'autre des choix induira une déperdition d'honneur personnel ou collectif. Lancelot choisit l'esquive. Ses écuyers passeront le pont; lui-même, malgré le danger, traverse à cheval la rivière "parfonde" et "ravineuse". Mais Neronneus retient l'écuyer chargé de l'écu de Lancelot, qu'il faut regagner "par forche d'armes” (\$ 8). Devant la honte que lui inflige Neronneus alors qu'il lui faisait à son insu "bonté et houneur", Lancelot n'a plus le choix : il repasse la rivière en suivant le même chemin que précédemment (!), et affronte victorieusement le gardien (§ 9-10). La coutume est appliquée avec un formalisme étroit (souligné par le jeu sur l'écu, métonymie de l'honneur) et un imperturbable sérieux. "Honte", "vilonnie" et "outrage", "houneur", "courtoisie” et "franchise” (\$ 9), déterminent les comportements. Lancelot s'efforce de respecter les formes : l'exigence d'une joute préalable au passage explique qu'il traverse une seconde fois la rivière, aussi absurde que cela paraisse. Il n'est pas concevable de modifier même la seule forme de la coutume sans annuler sa portée.

La référence à la norme sert une combinatoire narrative : la coutume étant un donné intangible, quelle attitude choisir pour ne pas déchoir ? Avec quelles conséquences, surtout lorsque le choix paraît absurde ? À partir de ces contraintes, le récit développe un nombre de schémas narratifs restreint, mais dont les variantes en fonction des circonstances sont presque infinies. Dans chaque situation, il y a au moins deux variables récurrentes : répondre ou contrevenir à l'injonction; préserver ou perdre l'honneur. Par exemple, en acceptant la joute contre Galaad pour venger l'honneur de Gaheriet abattu sous ses yeux, Yvain obéit à une norme, mais prend le risque d'un déshonneur personnel s'il est vaincu, ce qui se produit en effet (v. I, t. IV, § 146). Le récit se contente rarement de combinaisons simples, et relance ordinairement le dilemme en multipliant les normes et les personnages impliqués. Les micro-récits que nous évoquions comme un trait stylistique saillant du Tristan en prose sont presque tous fondés sur des combinaisons de ce type, dont on trouvera ci-après d'autres exemples. "Ouvert" du point de vue des possibles narratifs, le roman est en fait très circonscrit du point de vue idéologique : chaque épisode illustre ou discute un aspect de l'idéologie aristocratique. Le récit semble n'avoir d'intérêt que pour le dilemme ponctuel auquel le personnage se trouve confronté sans cesse, et son écriture même est fondée sur la mise en question permanente de l'honneur aristocratique : on pourrait parler à son propos d'une écriture du soupçon et de la preuve. De très nombreux épisodes mettent en doute la noblesse de tel chevalier incognito, ou exaltent au contraire la valeur qui transparaît au premier regard ${ }^{22}$.

22. Cf. v. I, t. I, chap. I, § $22:$ La dame ... dit bien a soi meïsmes quant ele l'ot asséz regardé qu'il est sans faille tot le plus bel chevalier que ele oucquez mes veïst, et c'il n'estoit outreement bon chevalier, se seroit le greigneur domage du monde. Sur l'incognito, cf. F. PLET, «Incognito et renommée. Les innovations du Tristan en prose », Romania, 120, 3-4, 2002, p. 406-431. 
Quant à la preuve (et à l'épreuve) de la valeur, il n'est qu'à relever le grand nombre d'occurrences du vocabulaire probatoire pour en illustrer l'importance ${ }^{23}$. La fragmentation du récit en cas d'écoles ( $c f$. infra) transforme ce trait idéologique en principe narratif, support d'une esthétique : le roman illustre en permanence l'idée que la noblesse doit être manifeste, que la dérogeance doit être dénoncée, et que les dons de la naissance doivent être corroborés par des preuves tangibles de vertu ${ }^{24}$.

La norme importe moins que le comportement qu'elle induit : même immotivée et contraignante, elle fait entrer en jeu l'impératif de préservation de l'honneur. En ce sens, la coutume est le signe de la prédétermination essentielle des chevaliers. Ainsi, lorsque Lancelot, Keu et Brandelis se trouvent, quelques paragraphes plus loin, à la croisée des chemins, Lancelot rappelle sentencieusement la règle :

« Tant sai je bien des coustumes du roiaume de Logres que, puis que cevalier viennent a une voie ki se part en deus u en trois, il se doivent tout maintenant departir. » (v. II, t. I, § 26)

Sa science chevaleresque est aussitôt approuvée comme convenant à la condition de chevalier errant :

«Sire, vous dites courtoisie et ce que chevaliers errans doit dire. » (ibid.)

Dans le rapport ainsi établi entre la courtoisie de Lancelot et une règle purement pratique, il faut comprendre que la courtoisie réside précisément dans la connaissance de la règle, perçue comme fondamentalement chevaleresque.

\section{Codes sociaux}

Figée en stéréotype narratif, la coutume n'est que l'une des facettes du code de conduite chevaleresque. Cette codification répond à un phénomène historique : Jean Flori a montré que la formation de la caste aristocratico-chevaleresque est achevée au XIII' siècle par l'élaboration d'un code propre à la caste, qui la constitue en groupe social alors que d'autres groupes ne possèdent rien de tel ${ }^{25}$. Du Lancelot en prose au Llibre de caballeria de Ramon Llull, la construction idéo-

23. Cf. v. II, t. IV, § 239: Mesire Tristrans ... avoit bien esprouvé et conneü que Helyans estoit uns des mieudres cevaliers k'il eüst piecha mais trouvé ...; ;. I, t. IV, §300: il est bien chevalier esprové et de valor et de bonté; Curtis, t. I, § 403 : l'en ne dreçoit onques escuz devant paveillons se ce ne fussent chevaliers aventureus qui les i fë̈ssent metre por eus esprover encontre toz ceus qui sor eus venroient.

24. Sur le rapport (problématique) entre naissance et vertu, $c f$. V. HonEmanN, « Aspekte des "Tugendadels" im europäischen Spätmittelalter », Literatur und Laienbildung im Spätmittelalter und in der Reformationszeit. Symposion Wolfenbüttel, 1981, hrsg. v. L. GRENZMANN und K. StackmanN, Stuttgart, 1984, p. 274-288, qui offre un bilan très complet des différentes "théories de la noblesse" depuis l'Antiquité tardive.

25. Cf. J. FloRI, L'essor de la chevalerie (XI'-XII ${ }^{e}$ s.), Genève, 1986. 
logique se précise tout au long du XIII ${ }^{\mathrm{e}}$ siècle. On pourrait s'attendre à ce que le Tristan en prose réitère ce "catéchisme" chevaleresque. Mais le rappel des devoirs de la chevalerie y est assez accessoire (par exemple v. II, t. III, § 202 ou VII, § 39$)^{26}$.

Pourtant, une série de règles précises structurent la sociabilité chevaleresque $^{27}$. Pour être reconnu comme un véritable chevalier, il faut "faire et dire que courtois", c'est-à-dire obtenir la reconnaissance de sa condition en fonction du respect de normes partagées. À chaque situation correspond une réaction appropriée et, corrélativement, le risque de commettre un impair. L'éventuelle dérogeance entraîne des sanctions réelles (joutes et combats) ou plus souvent symboliques, de l'ordre d'une déperdition d'honneur. On retrouve donc l'honneur au fondement de l'appareil normatif, valeur suprême, étalon des normes qui déterminent les comportements valides et invalides. Les usages courtois sont garants de l'honneur, dont ils démontrent en retour la fonction normative ${ }^{28}$. Le maintien d'un honneur qui croît et décroît sans cesse dépend de la volonté de l'individu ${ }^{29}$. Fondement de l'ethos chevaleresque, l'honneur vaut plus que la vie même, mais se révèle éminemment relatif car soumis à l'opinion commune ${ }^{30}$. Parce que l'honneur est difficile à définir, le texte se concentre essentiellement sur sa dimension pratique : puisqu'il s'agit de se comporter comme il convient, qu'est-ce qu'une situation, un acte, une parole honorables ou déshonorants? Quels critères normatifs permettent d'en juger ? Occupé à détailler les conditions concrètes de l'honorabilité, le récit se disperse en une collection de points d'honneur : ainsi s'expliquent, selon nous, la fragmentation et la stéréotypie du récit dans le Tristan en prose, et l'inscription d'une tendance didactique dans le texte littéraire. Si le Tristan en prose n'est assurément pas le seul roman de chevalerie à s'intéresser à l'honneur comme fondement de l'action, il se distingue - nous

26. Rien de comparable au «miroir de chevalerie » du Lancelot (E. Kennedy et F. MosÈs éd., Paris, 1991, p. 396-412).

27. Sur quelques-unes des formes de ce code, $c f$. M.-L. CHÊNERIE, Le chevalier errant dans les romans arthuriens en vers des XII ${ }^{e}$ et XIII' siècles, Genève, 1986, p. 282 sq., 312 sq. (combat), 521 sq. (l'hospitalité), etc. $C f$. p. 299 : «L'aventure paraît offrir à chacun l'occasion d'une adhésion personnelle, intime, autonome, à un code de valeurs où peu à peu doit se reconnaître tout un groupe. »

28. Cf. J. PitT-Rivers, op. cit., p. 10 : « [1'honneur] réunit dans sa définition la motivation du sujet et le jugement d'autrui, contribuant ainsi à la formation de normes. En même temps, il permet de saisir l'impact de ces normes sur la conscience des individus qui calculent leur bilan personnel d'honneur selon leur sensibilité de caractère. »

29. Cf. J. Pitt-Rivers, «La maladie de l'honneur », dans M. Gautheron dir., L'honneur. Image de soi ou don de soi : un idéal équivoque, Paris, 1992 (Autrement. Série "Morales", 3), p. 22-36: "L'essence de l'honneur, c'est la volonté » qui impose de se mettre en péril pour relever le défi et réparer l'affront (p. 25). $C f$. Curtis, I, § 290-1 : le très jeune Tristan se montre digne d'être adoubé en justifiant son hasardeux combat contre le Morholt par la nécessité d'acquérir l'honneur, ou de mourir dans la tentative.

30. $C f$. v. II, t. VIII, $\S 3$ : Galaad s'étonne de ce qu'un de ses adversaires puisse se dire honni et avilié d'avoir été vaincu. Pour le chevalier celestiel qui méprise la "doxa" de la gloire terrestre, il s'agit d'un honneur mal placé. Sur le concept d'honneur chrétien (fondé moins sur la Fortitudo que sur l'Humilitas), cf. A. von MüLler, Gloria bona fama bonorum. Studien zur sittlichen Bedeutung des Ruhmes in der frühchristlichen und mittelalterlichen Welt, Husum, 1977. 
semble-t-il - par la remarquable fréquence de la question au fil du récit. Il suffit d'ouvrir le roman au hasard pour mesurer l'ampleur du phénomène : «Jamais jour de ma vie n'avrai hounour! » (v. II, t. V, § 238), s'écrie le vaincu à la joute, quand les personnages les plus magnanimes ${ }^{31}$ ne songent qu'à maintenir ou acroistre leur honneur (v. II, t. V, § 219 et 220). Les chevaliers du Tristan en prose n'ont, littéralement, que ce mot à la bouche.

Les règles du point d'honneur n'ont rien d'inaltérable, mais s'élaborent tout au long du roman dans des «études de cas », où l'on recourt à un appareil normatif supposé connu et partagé par les personnages du fait de leur appartenance à la caste aristocratique. Un passage de la v. II, t. IX, § 7-8, illustre cette littérature du point d'honneur. Artus le Petit provoque Galaad à la joute sous les yeux de Palamède. Désarçonné, il s'acharne à le poursuivre pour réparer l'offense. S'engage alors un débat entre Palamède et Artus :

Palamidés [...] li dit : «Or savés vous bien conment li cevaliers jouste ! Se vous ne volés mourir u avoir greigneur honte que vous avés eüe, laissiés atant ester, car contre lui ne porriés vos durer en nule maniere ! Et s'il bien vausist, il vous eüst mort! Mais il le fist plus par sa courtoisie que par deboinaireté qu'il trouvast en vous !» Artus [...] li respont : «S'il est mieudres cevaliers de moi, ce connistrai je bien au ferir de l'espee !» [...] - «Certes, fait Palamidés, vous n'estes pas si courtois conme vous deüssiés estre, si vous dirai pour coi. Vous estes boins cevaliers et preus, si devriés estre courtois ; et vous estes feus et outrageus! Par l'envie que vous avés sus les boins cevaliers, les asailliés vous, et quidiés que ce soit courtoisie ! Certes, se cil qui de ci s'en vait eüst vostre coustume, qui est li miudres cevaliers du monde, si feroit il mout a blasmer ! » À ces paroles respont Artus li Petis : «Vous ne me devez pas blasmer se je me vois essaiant a vous et as autres preudommes, car jentiex hom et joveneciaus je sui, nouviaus cevaliers, si ai mestier de los et de pris, se je le puis conquerre; ne cevaliers ne doit refuser jouste d'autrui ne reposer, mais faire en sa joveneté cose dont il soit loés en sa viellece ! - «Çou est voirs, fait Palamidés. Mais toutevoies ne doit faire vilenie pour riens, puis qu'il est cevaliers !» (IX, § 8)

La sentence déterminante revient à Palamède : "Vous n'estes pas si courtois conme vous deüssiés estre." Cette phrase, qui met en question l'ethos aristocratique, est fréquente dans le Tristan en prose ${ }^{32}$. Elle introduit toujours une argutie sur les convenances : celui à qui elle s'adresse a dérogé à ce qu'on attend de lui en fonction d'une définition partagée de la courtoisie. On attend du personnage qu'il sache «ce qui se fait » et on le lui rappelle au besoin, selon le principe de régulation permanente des normes.

31. C'est-à-dire poussés par la magnanimitas : la conscience d'avoir une "grande âme", assortie de la nécessité de s'en montrer digne. Le Tristan en prose (à la suite de divers traités moraux), traduit cette qualité par le vocable haut cuer (v. II, t. II, $\S 9 ; c f$. trad. anonyme (XIII ${ }^{\mathrm{e}} \mathrm{s}$.) de la Formula honestae vitce de Martin de Braga (vi s.), éd. E. IRMER, 1890, v. 398-410). La magnanimitas est une manifestation de la vertu de la Fortitudo, fondatrice de l'existence chevaleresque. $C f$. notre thèse, p. 567-575.

32. Cf. v. II, t. II, §197-8: débat entre Palamède et une demoiselle sur la nature de la courtoisie ; v. II, t. III, $\S 196$ : stigmatisation d'une importunité ; v. II, t. VII, $\S 48$ : au sujet du trop fier Gauvain : «or saciés bien que tout li cevalier ne sont mie courtois. » 
Comme pour la coutume, on remarque que le débat se déplace rapidement des circonstances au contexte axiologique plus large. Selon Palamède, "courtoisie", "deboinaireté", "honte", "outrage" et "vilenie" sont en jeu. Artus se place également sur ce plan en invoquant "preudomie", "jentillesce", "los" et "pris", soit "valeur individuelle", "nature aristocratique" et "réputation". Il ne s'agit plus tant de savoir si le combat aura lieu ou non, que d'établir en quoi consiste, d'un point de vue pratique et relativement à cette situation précise, la notion même de courtoisie. Obtenir d'affronter Galaad envers et contre tout est bien un point d'honneur.

Le discours des deux chevaliers est innervé par des tournures évaluatives et normatives ${ }^{33}$. L'honneur varie en degrés, amenant un possible changement d'état de la personne. Il fait donc l'objet d'une évaluation sur une échelle graduée : "il le fist plus par... que par...", "vous n'estes pas si courtois conme...", "boins chevaliers / feus et outrageus", "mieudres cevaliers", "li miudres cevaliers du monde." Le discours évaluatif se lit encore dans le "blasme" (qui appelle le "los" et le "pris"), dans l'antithèse "joveneté / viellesce" (puisque "joveneté" est en rapport direct avec "jentillesce"), dans la nécessité qu'il y a à "soi essaier", enfin dans l'opposition entre "chevalerie" et "vilenie". Mais dans la balance de l'honneur, toutes les pièces ne sont pas du même aloi, aussi faut-il invoquer la norme de référence, à l'aide de la modalité du devoir: "vous n'estes pas si courtois conme vous deüssiés estre", "si devriés estre courtois", "se cil... eüst vostre coustume..., si feroit il mout a blasmer", "vous ne me devez pas blasmer", "si ai mestier de...", "chevalier ne doit refuser jouste..., mais faire...", "toutevoies ne doit faire vilenie..." Si le "devoir" est toujours un "devoir-être" et un "devoir-faire", l' "être", le "faire" ou le "devoir" se subordonnent au "savoir" - "Or savés bien conment li cevaliers jouste!" - qui détermine la compétence évaluative des personnages et motive leurs choix. C'est un défaut de "savoir" (une évaluation imparfaite) et conséquemment une méprise quant au "devoir", que stigmatise Palamède : "et quidiés que ce soit courtoisie ${ }^{34}$."

On remarquera en particulier l'énoncé de "lois" de la chevalerie, qui appuient de part et d'autre le propos :

- «cevaliers ne doit refuser jouste d'autrui »

- « [cevaliers] ne doit faire vilenie pour riens, puis qu'il est cevaliers. »

33. Cf. Ph. Hamon, Texte et idéologie, Paris, 1984, chap. I, « Texte et idéologie : pour une poétique de la norme ». La dimension idéologique d'un texte romanesque se lit dans des «foyers normatifs » (p. 20), où le récit est mis en perspective par un évaluateur (narrateur ou personnage), en fonction de normes. Il s'agit de classer propos et actes selon des critères d'intensité (positivité ou négativité, réussite ou échec, conformité ou déviance, validité ou invalidité, etc.) ou de les modifier par une modalité qui exprime la compétence normative de l'évaluateur (croire, vouloir, pouvoir, savoir, devoir, falloir). "Partout où il y a "intérêt" d'un sujet impliqué dans une relation médiatisée au monde, [...] il y aura norme implicitement convoquée. » (p. 38).

34. Le savoir sur l'autre est un élément important de la distinction, en ce qu'il permet d'établir les hiérarchies. Il importe au chevalier de connoistre autrui, éventuellement dans l'affrontement physique: "Sire cevaliers, nous nous sommes ensamble combatu une grant piece et tant avons ja fait que vous connissiés moi et je vous. » (v. II, t. I, § 56). 
Ces "lois" rencontrent un consensus relatif : "Çou est voirs", reconnaît Palamède. Nous sommes bien en présence d'un code normatif informel, relevant d'un savoir et d'une pratique communs à tous les chevaliers ${ }^{35}$. Informel ne signifie pas inopérant : la prégnance du discours normatif dans le détail même de l'écriture du Tristan en prose se mesure fort bien dans cette avalanche d'injonctions, qui toutes définissent l'ethos aristocratique. Sans aucun doute, noblesse oblige, au sens le plus littéral du terme.

Le point essentiel de ce type de débat est que les diverses normes invoquées sont à la fois valides et contradictoires. Aucun choix ne s'impose d'emblée comme le bon. Il y a donc une forte orientation normative du discours, mais jamais imposition d'une norme. En témoignent des épisodes d'une complexité qui confine à l'absurde, et qui relèvent clairement de la relativisation du code de conduite chevaleresque, si ce n'est de l'auto-parodie. Ainsi de ces péripéties du t. I de la v. I, chap. XII, § 18-31 : Lancelot, Keu, Bliobléris et un chevalier de rencontre se disputent la demoiselle qui accompagne ce dernier. Keu la conquiert le premier, mais Lancelot la lui conteste, arguant d'une curieuse "loi" stipulant qu'une demoiselle conquise en présence d'autres chevaliers appartient à tous les présents. Il demande à Keu de la lui céder par cortoisie. Keu refuse : s'il ne la défend pas les armes à la main, il y perdra son honneur. Lancelot l'abat sans difficulté, mais c'est au tour de Bliobléris de "réclamer sa part." Or Lancelot ne peut se battre contre son cousin et son pair à la Table Ronde sans déroger. Il choisit malgré tout cette solution, pour mettre Bliobléris à l'épreuve. Le choc est terrible, les chevaux sont tués, les deux adversaires évanouis. Revenu à lui, Lancelot quite la demoiselle à Bliobléris. Ce dernier accepte d'abord, car un chevalier ne saurait refuser une demoiselle. Mais il la refuse d'autre part, puisqu'il ne l'a pas gagnée ! Lancelot décide donc de laisser la demoiselle libre de son choix : après tout, une autre "loi" interdit de faire force à une femme... Celle-ci choisit de revenir à son premier protecteur, déclarant qu'elle a perdu assez de temps en compagnie de si tortueux individus. Lesquels, en l'absence de chevaux, quittent la scène, penauds, sur les roussins de leurs écuyers. Tout au long de l'épisode, le lecteur est laissé juge de la pertinence des "lois" invoquées. La seule norme incontestable est l'obligation de ne laisser sous aucun prétexte s'amoindrir l'honneur.

Mais comment conserver et accrôtre l'honneur sans trébucher dans l'écheveau des obligations, dont le nombre et la complexité mettent en eux-mêmes l'honneur en péril ? Dans de telles conditions, on risque en permanence de déroger. Les relations entre chevaliers sont régies par deux postulats non formulés : d'une part, il existe en toutes circonstances des règles susceptibles d'être transgressées ; d'autre part tout acte de revendication de l'honneur implique potentiellement une telle transgression, en ce qu'il menace l'honneur d'autrui. Les interlocuteurs ne se font pas faute de stigmatiser ces transgressions, réelles ou supposées. Le texte affiche une évidente délectation à multiplier jusqu' au vertige les injonctions normatives contradictoires.

35. Nous avons proposé dans notre thèse une typologie de ces "lois" (p. 739-751). Elles touchent tous les domaines de l'existence chevaleresque (combats, conversations, rencontres, séparations, rapport avec les femmes, etc.) Don Quichotte s'enorgueillira encore de les connaître, alors que Sancho, roturier et écuyer de fortune, les ignore. 
Cet aspect est ignoré des traités didactiques, auxquels échappe la dynamique de l'existence chevaleresque. L'Ordene de chevalerie ou le Roman des Eles (K. Busby éd., 1984) définissent une éthique et des valeurs; les "traités des manières" édictent des préceptes concrets. Le roman se fait, quant à lui, traité de casuistique aristocratique, où les micro-récits stéréotypés mettent en scène et font jouer les unes contre les autres les règles de la chevalerie. Cette ritualisation est perçue comme la mise en lumière de la conscience qu'a chacun de sa valeur, sans cesse jugée à l'aune du réseau normatif. En mettant en scène la complexité des normes aristocratiques, le roman les élève au rang d'art de vivre : la connaissance des usages n'est pas à la portée de tout un chacun.

On peut donc envisager le récit comme la juxtaposition de «cas d'école». Les paragraphes $\S 145-8$ du t. VIII (v. II) fournissent encore un exemple de leur degré de complexité. Un violent combat oppose une fois de plus Palamède à Tristan. Arrive Blyoblerys qui, sans les reconnaître, suppose au vu de leur "grant hardement" qu'ils appartiennent tous deux à la Table Ronde. Il souhaite séparer les combattants, conformément à la règle qui interdit, dans la mesure du possible, l'affrontement de deux membres de la Table Ronde [norme 1]. Mais il se heurte d'emblée à un autre impératif :

[norme 2] Il les departiroit trop volentiers s'il pooit, en tel maniere que l'ounour de l'un et de l'autre i fust sauvee. (VIII, § 145)

Il choisit d'interrompre le combat sous prétexte de demander leur nom aux combattants. Tristan lui reproche vertement d'avoir manqué à la courtoisie en intervenant dans les affaires d'autrui, sans respecter l'incognito :

[norme 3] «A vous qu'en apartient? fait mesires Tristrans. Ce n'est pas courtoisie d'enquerre les cevaliers estranges qui i sont, car aucunes fois vont il querant aventures qu'il ne vauroient pour riens c'on les conneüst ! (VIII, § 146)

Blyoblerys manifeste son assentiment, mais en appelle à une coustume :

[norme 4] Sire, fait Blyoblerys, vous poés bien dire voir de ceste cose. Mais il est coustume que ${ }^{36}$, quant uns cevaliers voit.II. bien preudomes ensamble par ire et par mautalent en une place, s'il nel connoist, et il est desirant de connoistre les pour la bonté k'il voit en aus, et il lour demande lor estre, nus ne le doit tenir a vilonnie. (ibid.)

Tristan accepte de révéler son nom, de même que Palamède. Or, Blyoblerys se trouve avoir une dette envers Palamède, qui l'a récemment sauvé de la mort. Fort de cette obligation [norme 5], le Sarrasin en difficulté demande à Blyoblerys d'intervenir en sa faveur auprès de Tristan. Celui-ci refuse la médiation. Palamède se jette alors à genoux et rend son épée. Ce geste, accompli devant un tiers qui

36. Faut-il comprendre : «il est habituel que» ou plutôt «il est prescrit que »? L'ambiguïté de la formulation signale la situation instable de la norme, entre conduite prescrite et conduite intériorisée. La seule référence à une ligne de conduite suffit en tout cas à provoquer l'assentiment de Tristan. 
n'ignore plus rien de l'estre de Tristan, interdit à ce dernier de passer outre à ce qui est encore une coustume de la Table Ronde (cette fois une prescription institutionnelle) :

[norme 6] Quant mesire Tristrans voit ceste aventure, il est trop iriés, si qu'il vauroit bien morir maintenant, car il ne haoit home si mortelment com il faisoit celui, si ne s'em puet vengier k'il n'en fust parjures et desloiaus por la compaingnie de la Table Reonde, car la coustume estoit tele que nus qui compains en fust ne pooit metre main sus cevalier puis qu'il li rendoit s'espee. (VIII, § 148)

Le poids des prescriptions spécifiques à l'élite de la chevalerie - la plupart de ces normes sont en fait des "lois" de la chevalerie - met en fin de compte un coup d'arrêt aux velléités de vengeance.

La casuistique vise à examiner dans la pratique le code de conduite de la chevalerie, et sert en même temps d'instrument de distinction. La "science" chevaleresque, fondée sur l'analyse de ces "cas d'école", permet de distinguer les "boins chevaliers" - ceux qui font les meilleurs choix dans l'appareil normatif des "mauvais" qui manquent de discernement ${ }^{37}$. Même si les lois de la chevalerie ne sont pas toujours formulées explicitement, leur validité est tacitement reconnue dans la possibilité de référence à un appareil normatif conçu comme un appareil distinctif $^{38}$.

\section{Conversation}

La casuistique prend vie dans la conversation qui roule sur un point du code, procédé qui n'est pas sans rappeler la casuistique amoureuse telle qu'elle se lit chez André le Chapelain ou dans le jeu-parti ${ }^{39}$. Nous ne nous consacrerons ici qu'à la conversation en tant qu'occupation mondaine, d'autant que la conversation amoureuse a déjà été largement explorée à propos du Tristan en prose ${ }^{40}$. La

37. Parmi les qualités courtoises, le discernement est hautement valorisé (V, § 89: mesire Tristrans, ki assés estoit percevans cevaliers). Sous le vocable "discrétion", il appartient aux valeurs de la distinction classique (Dictionnaire raisonné de la politesse..., op. cit., article « Discrétion»). Son équivalent médiéval est la Prudence recommandée au chevalier par nombre de traités moraux.

38. Il faudrait traiter encore de tout ce qui relève de la régulation des normes et de la capacité à s'en affranchir. Ce rôle est dévolu en particulier à Dinadan : $c f$. (au sein d'une large bibliographie), A. Berthelot, «Dynadam le chevalier non conformiste », Conformité et déviances au Moyen Âge. Actes du $2^{e}$ colloque international de Montpellier (25-27 nov. 1993), Montpellier, 1995 (Cahiers du CRISIMA, 2), p. 33-41 et plus récemment F. ZAMBON, «Dinadan en Italie », Comedy in Arthurian Literature, éd. by K. Busby and R. Dalrymple, Woodbridge, 2003, p. 153-163 ou B. WAHLEN, «Le Bon Chevalier sans Peur, Brunor, Dinadan et Drian : un lignage détonnant!", communication au $3^{\mathrm{e}}$ colloque international de l'Université de Rennes-II, 1314 octobre 2005, à paraître.

39. Cf. A. Le Chapelain, Traité de l'amour courtois, Cl. Buridant trad., Paris, 1974, L. II, chap. VII.

40. Cf. D. Demartini-Franzini, Miroir d'amour, miroir du roman. Le discours amoureux dans le "Tristan en prose", Paris, Champion, 2006, p. 249-261. 
conversation a toujours été conçue comme une pratique aristocratique. Certes, il n'existe pas de traités de conversation médiévaux comparables aux traités classiques $^{41}$, mais l'évident plaisir qu'éprouve le Tristan en prose à mettre en scène l'échange de parole - on a parlé à ce sujet d' « inflation rhétorique ${ }^{42}$ » - laisse penser que la littérature a pu, là encore, jouer ce rôle. La conversation courtoise n'a qu'un objet : l'excellence dans ses divers et complexes mécanismes, en fonction de la satisfaction ou de la dérogeance aux normes courtoises. Elle adopte elle-même des règles dont on se bornera à souligner quelques aspects.

Qu'il s'agisse d'obtenir d'autrui des renseignements, ou d'énoncer ce qu'il faut faire et être pour correspondre au mieux à l'idéal courtois, l'échange est toujours évaluatif et normatif ${ }^{43}$. Or, ces interactions peuvent aisément être perçues comme des atteintes à l'intégrité de l'individu. La conversation apparaît alors comme le substitut non violent du combat. Comme l'escremie, elle connaît des conventions stéréotypées : celles de la politesse, qui vise à imposer des normes aux interactions sociales et à prévenir, à apaiser ou à gérer les conflits ${ }^{44}$. La politesse s'exprime dans de très fréquentes formules d'atténuation, de prière, de mise à distance ou de virtualisation du propos, même dans les situations les plus tendues. Ainsi, on sollicite de pouvoir se battre en tournures élégantes; se relevant après avoir été abattu par Neronneus, Keu d'Estraus tient ce discours :

«Sire cevaliers, je voi bien que m'avez abatu. Mais pour ce se vous m'avés abatu, ne m'avés vous pas mené a outrance. S'il vous plaist et vous avez talent de bataille, je sui apareilliés de combattre a vous. » (v. II, t. I, § 5)

Cependant, étant admis que l'échange policé constitue la règle, des représailles graduées sont admises en cas de manquement. "A vous qu'en apartient ?” rétorque brutalement Tristan à Blyoblerys, rendant compte de son indignation face à l'importunité discourtoise d'un tiers.

41. L'intérêt pour le «bien parler » comme indice de distinction remonte à la morale et à la rhétorique antiques. Au Moyen Âge ce type de réflexion se trouve, entre autres, chez AlBERTANo DA Brescia, Ars loquendi et tacendi, (T. Sundby, éd., Copenhague, 1869) ou Brunet Latin, Le livre du Trésor, II, (F. J. CARMody éd., Berkeley, 1948).

42. Cf. A. BerTHELOT, «L'inflation rhétorique dans le Tristan en prose », dans D. BusChINGER éd., Tristan et Iseut, mythe européen et mondial. Actes du colloque des 10, 11 et 12 janvier 1986, Göppingen, 1987, p. 32-41.

43. Remontrance : $c f$. supra, débat entre Artus le Petit et Palamède ; demande de renseignements : $c f$. infra, questions sur l'identité ; échange de vues : III, § 223-224 : comment définir le boin cevalier ? Conseil : I, § 146 : Keu appelé à résoudre un cas épineux relatif à un serment.

44. Cf. C. Kerbrat-Orecchioni, Les interactions verbales, t. II et III, Paris, 1992-1994. La politesse régit le discours dans le but de préserver l'harmonie des relations interpersonnelles. L'individu est amené à protéger son "territoire", que l'interaction verbale menace nécessairement. Ainsi, la prière, l'ordre, le reproche, l'insulte, la question indiscrète, etc. mettent l'interlocuteur en difficulté ; l'offre, la promesse, l'excuse ou le remerciement mettent le locuteur en position de faiblesse. Il faut à la fois ne pas «perdre la face » et ménager autrui : la politesse est travail de mise en scène des relations sociales. Soit on évite de produire des énoncés menaçants (atténuation de la prière, de l'ordre...), soit on produit des énoncés valorisants (compliment, remerciement, vœu...). 
Un usage adroit du compliment ouvre la voie à une demande délicate quant à l'identité de l'adversaire (car connaître l'identité et l'estre permet d'établir un classement des individus ${ }^{45}$ ):

« Je di bien en moi meïsmes que vous estes sans doute li mieudres cevaliers et li plus fors que je onques trouvaise [...]. Et pour ceste cose vauroie je mout volentiers, s'il vous plaisoit, savoir ki vous estes.» (v. II, t. I, § 13)

Les plus "discrets" des chevaliers ne sont d'ailleurs pas dupes de ces formalités, immédiatement identifiées comme appartenant aux normes du discours bienséant, auxquelles on oppose une modestie de bon ton :

[Tristan] «Por la bonté et pour la proueche que je ai en vous trouvee [...] je vous pri, tant com porroie proiier chevalier, que vostre nom me diés [...]» - [Lancelot] « Vous me donnés, ce m'est avis, grant los et grant pris de chestui fait. Ensi est des coses du monde que li sages cevaliers set tous dis son anemi blasmer par beles paroles ! [...] Et quant vous ce me requerés, se je refusoie vostre proiiere, bien le me devroit on atourner a orgoeil et a felonnie. » (v. II, t. III, $\S 255-6)$

Enfin, ces demandes comportent souvent des formules de contrainte polie ${ }^{46}$, prières stéréotypées qui sont en réalité des injonctions atténuées, auxquelles il est cependant interdit de se soustraire :

«Je vous pri, tant com porroie proiier chevalier...» (v. II, t. III, § 255)

«Se Diex vous doinst boine aventure, sire cevaliers, et se Diex vous doinst joie de la riens que vous plus amés, dites moi conment vous avés non, pour savoir se je vous poroie connoistre. - Si m'aïst Dieus, fait Boorch, vous m'avés tant conjuré que je le vous dirai. » (v. II, t. V, § 230)

La prière "au nom de l'objet du plus grand amour", assez fréquente, suppose un assentiment obligatoire (même de mauvaise grâce). Il s'agit d'une norme du discours poli, qui permet de fléchir l'interlocuteur à l'aide une procédure codifiée et acceptée de part et d'autre, selon un principe exactement semblable à celui des coutumes institutionnelles et des lois immanentes de la chevalerie ${ }^{47}$.

La maîtrise de la parole est un atout : le roman souligne les dons langagiers de certains personnages ${ }^{48}$. Le chevalier s'assure ainsi le contrôle de la situation,

45. Sur cette importante question, $c f$. F. PLET, Les noms propres dans le "Tristan en prose", Thèse Paris-X, 2000 (à paraitre).

46. La politesse est aussi «forme socialisée de la violence » puisqu'elle force le sujet à rabaisser ses prétentions et contraint autrui à la réciprocité. Selon R. DHoQuors dir., La politesse. Vertu des apparences, Paris, 1991 (Autrement, Série « Morales », 2), p. 130, il y a « corrélation entre intensité de la violence sociale et importance des codes de savoir-vivre. »

47. Cette injonction relève des énoncés contraignants bien connus (dons en blanc, serments, conjurations). $C f$. la thèse de $\mathrm{S}$. COOPER-DenIAU, Le motif du don contraignant dans la littérature arthurienne du XII et XIII ${ }^{e}$ siècle (1150-1250), Paris-IV Sorbonne, 2000.

48. Cf. II, § 22 : Hestors de Marés, ki plus estoit un poi emparlés que li autre [...] ; III, $\S 229$ : Keu et Dinadan: Ambedoi estoient boin cevalier des armes et boines paroles avoient merveilleusement. 
et acquiert la possibilité de lui imposer sa propre orientation normative. La conversation élégante s'inscrit dans le cercle vertueux de la prescription normative : bien parler consiste à respecter les normes interactionnelles de la société aristocratique ; de cette manière, l'art de la conversation signale l'homme de bien; en retour, il lui confère l'autorité nécessaire pour prendre part à la casuistique qui régule les normes de la bonne société.

Le Tristan en prose se distingue par le poids écrasant du discours normatif : autant (et peut-être plus) que d'autres romans contemporains, il est lisible comme un "roman de l'aristocratie". La distinction y est décrite comme la soumission consentie à des règles dont le respect signale, au sein de la communauté aristocratique, l'individu particulièrement digne d'honneur. Texte littéraire et discours normatif se confortent l'un l'autre : le respect ou la transgression des normes sont sentis comme fournissant matière à récit. En effet, les "lois de la Table Ronde" sont presque l'unique objet du récit. Notons que ces "lois chevaleresques" n'étaient pas toujours informelles : une vingtaine d'entre elles ont été regroupées par un lecteur du $\mathrm{Xv}^{\mathrm{e}}$ siècle, en un court recueil qui rappelle les statuts des ordres de chevalerie du Moyen Âge tardif ${ }^{49}$. Don Quichotte, lecteur burlesque et pathologique (?), ne pouvait rêver mieux, dans sa dévotion envers les usages des chevaliers d'antan... Le formalisme tatillon de cette littérature peut surprendre par sa futilité apparente. Ce serait pourtant faire erreur de croire qu'il s'oppose à l'esprit aristocratique. Il en exprime au contraire une tendance profonde: la noblesse dérive de la contrainte qu'elle s'impose, si inutile soit-elle en apparence. En son temps, Saint-Simon ne dira-t-il pas comment, dans la vertigineuse différence entre un fauteuil et une "forme" (un tabouret), se lisait un garant essentiel de la dignité ducale ${ }^{50}$ ? Sans doute, l'étiquette n'existe pas encore pour les contemporains du Tristan en prose. Mais les conditions de son existence sont déjà réunies dans la mentalité aristocratique.

\section{Olivier LINDER}

\section{Aspects du discours normatif dans le Roman de Tristan en prose (coutumes, codes sociaux, conversation)}

Le lecteur moderne du Tristan en prose est frappé par la fréquence des prescriptions relatives au code de conduite chevaleresque et courtois : le chevalier existe en tant que tel à la seule condition de "faire ce qu'il doit faire" et de ne pas déchoir.

49. Cf. R. TrachsLer, «Les Lois de la Table Ronde», Studi Francesi, 40, 1996, p. 56785. Malgré leur caractère très général, il est possible que certaines de ces lois proviennent d'une version du Tristan en prose (la loi n ${ }^{\circ} 18$ évoque les deux épées de Palamède).

50. Cf. Saint-Simon, Mémoires (Y. Coirault éd., Paris, 1990), I, p. 147-9. Cf. N. Élias,

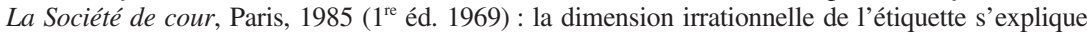
par sa fonction distinctive. Le moindre détail assure la préséance des courtisans les uns sur les autres en concrétisant l'honneur qui leur est dû. Ainsi, l'étiquette n'est pas sentie comme une contrainte mais comme la manifestation même de l'ethos aristocratique, une "auto-représentation » où, «chacun se distinguant de l'autre », et « des personnes étrangères au groupe », tous « s'administr[ent] la preuve de la valeur absolue de leur existence» (p. 97). 
Le phénomène concerne essentiellement la sociabilité aristocratique, c'est-à-dire les normes qui régissent les rapports entre personnes de distinction. Ces normes, le plus souvent fictives, sont l'équivalent littéraire de normes sociales avérées (l'éthique chevaleresque, la politesse, les usages distingués...). Leur omniprésence les mue en principes narratifs, le récit se concentrant sur l'analyse des interactions entre les diverses normes mises en jeu par une situation donnée. On peut mesurer ainsi à quel point la vision aristocratique du monde, fondée sur l'idée de contrainte valorisante ("Noblesse oblige"), conditionne profondément l'écriture même du roman de chevalerie.

Aristocratie - Normes sociales - Distinction - Coutumes - Conversation

\section{Some Aspects of the Normative Discourse in the Prose Tristan (customs, social codes, conversation)}

To the modern reader of the Prose Tristan, the frequence of instructions derived from the courtly and chivalric code is striking : the knight only exists as a knight as long as he "does what he must do" and does not fall from rank. This observation particularly applies to aristocratic sociability, that is the norms applied to relashionships between people of rank and distinction. These norms, often conveyed through fiction, are the literary equivalent of otherwise known social norms (chivalric ethics, courtliness, distinguished manners...). By being present everywhere, they become actual narrative elements - the narrative itself being narrowed to an analysis of the possible interactions between the various norms which may bear on a given situation. It can then be shown how much the aristocratic world vision, based on the idea of constraint as a self-enhancing value ("Noblesse oblige !"), deeply informs the very writing of the chivalric novel.

Aristocracy - Social Norm - Distinction - Manners - Conversation 
